\title{
FULL LIKELIHOOD INFERENCES IN THE COX MODEL: AN EMPIRICAL LIKELIHOOD APPROACH
}

\author{
JIAN-JIAN REN ${ }^{1}$ AND MAI ZHOU ${ }^{2}$ \\ University of Central Florida and University of Kentucky
}

\begin{abstract}
For the regression parameter $\beta_{0}$ in the Cox model, there have been several estimators constructed based on various types of approximated likelihood, but none of them has demonstrated small-sample advantage over Cox's partial likelihood estimator. In this article, we derive the full likelihood function for $\left(\beta_{0}, F_{0}\right)$, where $F_{0}$ is the baseline distribution in the Cox model. Using the empirical likelihood parameterization, we explicitly profile out nuisance parameter $F_{0}$ to obtain the full-profile likelihood function for $\beta_{0}$ and the maximum likelihood estimator (MLE) for $\left(\beta_{0}, F_{0}\right)$. The relation between the MLE and Cox's partial likelihood estimator for $\beta_{0}$ is made clear by showing that Taylor's expansion gives Cox's partial likelihood estimating function as the leading term of the full-profile likelihood estimating function. We show that the log fulllikelihood ratio has an asymptotic chi-squared distribution, while the simulation studies indicate that for small or moderate sample sizes, the MLE performs favorably over Cox's partial likelihood estimator. In a real dataset example, our full likelihood ratio test and Cox's partial likelihood ratio test lead to statistically different conclusions.
\end{abstract}

Keywords and Phrases: Right censored data; empirical likelihood; maximum likelihood estimator; partial likelihood; profile likelihood.

\section{March 27, 2009}

\footnotetext{
${ }^{1}$ Corresponding author; the author's research was partially supported by NSF Grant DMS-0604488; Mailing Address: Department of Mathematics, University of Central Florida, Orlando, FL 32816, USA; E-mail Address: jren@mail.ucf.edu.

${ }^{2}$ The author's research was partially supported by NSF Grant DMS-0604920; Mailing Address: Department of Statistics, University of Kentucky, Lexington, KY 40506, USA; E-mail Address: mai@ms.uky.edu.
} 


\section{INTRODUCTION}

Since Cox (1972), the following Cox's proportional hazards regression model has become one of the most widely used tools in analyzing survival data:

$$
\lambda(t ; z)=\lambda_{0}(t) \exp \left(z^{\mathrm{T}} \beta_{0}\right)
$$

where $Z$ is a $p$-dimensional vector of covariates, $\beta_{0}$ is the regression parameter, and $\lambda(t ; z)$ is the conditional hazard function of continuous random variable (r.v.) $X$ given $Z=z$ with $\lambda_{0}(t)$ as an arbitrary baseline hazard function. Suppose that $\left(X_{1}, Z_{1}\right), \cdots,\left(X_{n}, Z_{n}\right)$ is a random sample of $(X, Z)$, and the actually observed censored survival data are

$$
\left(V_{1}, \delta_{1}, Z_{1}\right),\left(V_{2}, \delta_{2}, Z_{2}\right), \cdots,\left(V_{n}, \delta_{n}, Z_{n}\right),
$$

where $V_{i}=\min \left\{X_{i}, Y_{i}\right\}, \delta_{i}=I\left\{X_{i} \leq Y_{i}\right\}$, and $Y_{i}$ is the right censoring variable with distribution function (d.f.) $F_{Y}$ and is independent of $\left(X_{i}, Z_{i}\right)$ or independent of $X_{i}$ given $Z=Z_{i}$. Then, Cox's partial likelihood estimator $\hat{\beta}_{c}$ for $\beta_{0}$ is given by the solution of equations:

$$
\varphi_{n}(\beta) \equiv n^{-1} \sum_{i=1}^{n} \delta_{i}\left(Z_{i}-\frac{\sum_{j=1}^{n} I\left\{V_{j} \geq V_{i}\right\} Z_{j} \exp \left(Z_{j}^{\mathrm{T}} \beta\right)}{\sum_{j=1}^{n} I\left\{V_{j} \geq V_{i}\right\} \exp \left(Z_{j}^{\mathrm{T}} \beta\right)}\right)=0,
$$

see Tsiatis (1981). In the past few decades, $\hat{\beta}_{c}$ has been considered as the standard estimate for $\beta_{0}$ in statistical literature. Efficiency properties of $\hat{\beta}_{c}$ were discussed by Efron (1977) and Oakes (1977). In particular, Efron (1977) examined the complete likelihood function that is parameterized through baseline hazard function $\lambda_{0}(t)$ in (1.1), and showed that Cox's partial likelihood function contains nearly all of the information about $\beta_{0}$, and $\hat{\beta}_{c}$ is asymptotically efficient. Using the counting process approach, the books by Fleming and Harrington (1991), and Andersen, Borgan, Gill and Keiding (1993) give a complete treatment of asymptotic theory and include many relevant references. We also refer to Cox and Oakes (1984), Therneau and Grambsch (2000), Kalbfleisch and Prentice (2002) for discussions and references on developments of the Cox model.

However, as pointed out in Cox and Oakes (1984; page 123), the efficiency results on $\hat{\beta}_{c}$ are only asymptotic, and for finite samples the loss in precision from using the partial likelihood can be rather substantial. It is well known and confirmed clearly by our simulation results (some of which are presented in Section 3) that the loss of efficiency can occur when, among other possible situations, the sample size is small or moderate, or $\beta_{0}$ is far from 0 . For instance, the numerical results presented in Table 1 of Kay (1979) show up to $18 \%$ loss of efficiency for $\beta_{0}=0.5$ with rather large sample size $n=1000$. It is also well known that in medical clinical trials, the sample size of survival data is often small or moderate. 
In literature, there have been some estimators for $\beta_{0}$ constructed based on various types of approximated likelihood, viz., the likelihood by grouping continuous model (Kalbfleisch and Prentice, 1973), the likelihood by discretizing the continuous Cox model (Bailey, 1984), etc. But none of these estimators has demonstrated small-sample advantage over Cox's partial likelihood estimator $\hat{\beta}_{c}$. With these in mind, a natural question would be: Does the actual maximum likelihood estimator (MLE) for $\beta_{0}$ (i.e., the MLE based on the exact complete or full likelihood) perform better for small or moderate samples? We do not know the answer to this question because up to now the actual MLE has not been given in the literature.

Using Poisson process arguments and parameterization via baseline hazard $\lambda_{0}(t)$, Efron (1977) showed that the complete or full likelihood function can be expressed as the product of Cox's partial likelihood function and a factor which involves both $\beta$ and observed data; see equation (3.10) of Efron (1977). This means that for finite samples, the inference based on the partial likelihood is not based on all the observed data in the sense that the partial likelihood is not the likelihood of observed sample (1.2); see discussions on page 559 of Efron (1977). But, in Efron's formula it is not obvious how to profile out nuisance parameter $\lambda_{0}(t)$ in order to obtain the actual MLE for $\beta_{0}$.

In this article, we derive the full likelihood function for $\left(\beta_{0}, F_{0}\right)$ in Cox model (1.1) with survival data (1.2), where $F_{0}$ is the baseline distribution corresponding to baseline hazard function $\lambda_{0}(t)$. Using the empirical likelihood parameterization (Owen, 1988), we explicitly profile out nuisance parameter $F_{0}$ to obtain the full-profile likelihood function for $\beta_{0}$ as well as the actual MLE for $\beta_{0}$.

Note that the key to achieving our results here is the combination of utilizing the Lehmann family properties and the empirical likelihood techniques. Although Cox's partial likelihood has been carefully studied in the past 35 years, the Lehmann family properties have not been used to derived the exact full likelihood function for the continuous Cox model (1.1). The empirical likelihoods have had a long history in survival analysis. Since Owen (1988), empirical likelihood has been developed as a powerful nonparametric inference approach, and many authors have applied it in survival analysis; see Owen (2001) and a review paper by Li, Li and Zhou (2005) for detailed descriptions and many references on this topic. As reviewed in $\mathrm{Li}$, $\mathrm{Li}$ and Zhou (2005) for works on the Cox model with right censored data, Pan (1997) obtained an approximated empirical likelihood function for $\left(\beta_{0}, \Lambda_{0}\right)$ in the Cox model (1.1), where $\Lambda_{0}$ is the cumulative hazard function of $\lambda_{0}$. By discretizing $\Lambda_{0}$, she showed that nuisance parameter $\Lambda_{0}$ is profiled out via the Breslow estimator (Breslow, 1974), and the resulting profile likelihood function for $\beta_{0}$ is the Cox's partial likelihood. This result was also obtained in Murphy and van der Vaart (2000), who referred their approach as empirical likelihood due to the use of point masses on the hazard function. However, one of the unsatisfactory features of the Breslow estimator is that the estimated 
hazard masses can exceed 1 (Kalbfleisch and Prentice, 2002; pages 116-117), in turn, the corresponding estimator for $\bar{F}_{0}$ can have negative values; see formula (2.10) on page 15 of Cox and Oakes (1984) for discrete distributions. Different from these existing works, our approach is based on the full likelihood function for $\left(\beta_{0}, F_{0}\right)$ in Cox model (1.1), which, when profiling out the nuisance parameter $F_{0}$ by discretizing, is different from the likelihood function parameterized via $\left(\beta_{0}, \Lambda_{0}\right)$. And our resulting MLE for $F_{0}$ is always a proper distribution function with values between 0 and 1 , while our resulting MLE for $\beta_{0}$ show favorable simulation performances over Cox's estimator $\hat{\beta}_{c}$.

The main materials in this article are organized as follows. Section 2 derives the full likelihood function for $\left(\beta_{0}, F_{0}\right)$ and the $\operatorname{MLE}\left(\hat{\beta}_{n}, \hat{F}_{n}\right)$ for $\left(\beta_{0}, F_{0}\right)$, where the relation between $\hat{\beta}_{n}$ and $\hat{\beta}_{c}$ is made clear by showing that Taylor's expansion gives Cox's partial likelihood estimating function $\varphi_{n}(\beta)$ as the leading term of the full-profile likelihood estimating function $\psi_{n}(\beta)$. Section 2 also shows that the log full-likelihood ratio has an asymptotic chi-squared distribution. Section 3 discusses computational issues and treatment of ties, and presents some simulation results which show that the MLE $\hat{\beta}_{n}$ performs favorably over $\hat{\beta}_{c}$ for small or moderate sample sizes, especially when $\beta_{0}$ is away from 0 . In Section 4 , we discuss a real dataset example, which demonstrates that in practical situation our full likelihood ratio test and Cox's partial likelihood ratio test may sometimes lead to statistically different conclusions. Some concluding remarks are included in Section 5 .

The findings in this article suggest that the MLE is preferred over Cox's partial likelihood estimator when sample size $n$ is small or moderate. This should not be a surprise since the MLE is based on all the observed data in the sense that it is based on the exact full likelihood of observed sample (1.2). In contrast, Bailey's estimator (Bailey, 1984) is based on an approximated likelihood function obtained by assuming a discrete baseline distribution $F_{0}$ (despite the fact that the Cox model assumes a continuous $F_{0}$ for continuous lifetime variables), thus his estimator is different from our actual MLE $\hat{\beta}_{n}$, and more importantly 'numerical studies suggest no small-sample advantage' (Bailey, 1984; page 734). Our other appealing findings here include: (a) Wilk's theorem holds for the log full-likelihood ratio of $\beta_{0}$; (b) the full likelihood function leads to the MLE jointly for $\left(\beta_{0}, F_{0}\right)$; (c) the computation for the MLE $\hat{\beta}_{n}$ is only slightly more complicated than Cox's partial likelihood estimator $\hat{\beta}_{c}$; (d) our method can be extended to deal with other types of censored data. The result of Wilk's theorem is of interest here because it has been established generally for parametric full likelihoods, but not generally for nonparametric or semiparametric full likelihood functions, while the Cox model (1.1) is a semiparametric model. The last point (d) is of particular interest because it is well known that the counting process approach is applicable to right censored data, but not complicated types of censored data, such as doubly censored data (Chang and Yang, 1987; Gu and Zhang, 1993), interval censored data (Groeneboom 
and Wellner, 1992), etc. However, the extension to other types of censored data requires additional and non-trivial work which will not be considered in this current paper.

While the main focus of this paper is the estimation of $\beta_{0}$ with small or moderate sample size $n$, it is worth noting that the MLE $\hat{F}_{n}$ for baseline distribution $F_{0}$ does not require any extension or approximation of the continuous proportional hazard model to discrete data; rather it is based on the full likelihood function with restriction of all possible candidates for the MLE of $F_{0}$ to those distribution functions that assign all their probability masses to observations $V_{i}$ 's and interval $\left(V_{(n)}, \infty\right)$. In contrast, there have been some competing methods to estimate $F_{0}(t)$ or the cumulative baseline hazard function $\Lambda_{0}(t)$ that require the use of discrete logistic model (Cox, 1972), or grouping continuous model (Kalbfleisch and Prentice, 1973), or discretizing continuous proportional hazard model to have approximated MLE (Breslow, 1974) in the context of counting process (Andersen and Gill, 1982), etc.. For detailed discussions and more references, we refer to Andersen, Borgan, Gill and Keiding (1993; Section IV.1.5) and Kalbfleisch and Prentice (2002; page 143).

\section{Maximum Likelihood Estimators}

For simplicity of presentation, this section considers the case that covariate $Z$ is a scaler rather than a vector, i.e., $p=1$ in (1.1). The generalization of our results to multivariate case with $p>1$ is straightforward and is summarized at the end of this section.

To derive the full likelihood function for $\left(\beta_{0}, F_{0}\right)$, we notice that under the assumption of Cox model (1.1), each $X_{i}$ has a d.f. that satisfies the following Lehmann family properties (Kalbfleisch and Prentice, 2002; page 97):

$$
\bar{F}\left(t \mid Z_{i}\right)=\left[\bar{F}_{0}(t)\right]^{c_{i}} \Leftrightarrow f\left(t \mid Z_{i}\right)=c_{i} f_{0}(t)\left[\bar{F}_{0}(t)\right]^{c_{i}-1}
$$

where $c_{i}=\exp \left(Z_{i} \beta\right)$ with $\beta=\beta_{0}, \bar{F}_{0}(t)=\left[1-F_{0}(t)\right]$ and $F\left(t \mid Z_{i}\right)$ is the conditional d.f. of $X_{i}$ given $Z=Z_{i}$, while $f\left(t \mid Z_{i}\right)$ and $f_{0}(t)$ are the density functions of $F\left(t \mid Z_{i}\right)$ and $F_{0}(t)$, respectively. We also notice that if $g(v, \delta \mid z)$ represents the conditional p.d.f. of $\left(V_{i}, \delta_{i}\right)$ given $Z_{i}=z$ for survival data $(1.2)$, we have $g(v, 1 \mid z)=f(v \mid z) \bar{F}_{Y}(v)$ and $g(v, 0 \mid z)=f_{\mathrm{Y}}(v) \bar{F}(v \mid z)$.

Thus, under the Cox model (1.1) with right censored survival data (1.2), the likelihood function of $\left(V_{i}, \delta_{i}\right)$ given $Z=Z_{i}, 1 \leq i \leq n$, is given by

$$
\prod_{i=1}^{n} g\left(V_{i}, \delta_{i} \mid Z_{i}\right)=\prod_{i=1}^{n}\left(f\left(V_{i} \mid Z_{i}\right) \bar{F}_{Y}\left(V_{i}\right)\right)^{\delta_{i}}\left(f_{Y}\left(V_{i}\right) \bar{F}\left(V_{i} \mid Z_{i}\right)\right)^{1-\delta_{i}}
$$

which under (2.1) is proportional to

$$
\prod_{i=1}^{n}\left(f\left(V_{i} \mid Z_{i}\right)\right)^{\delta_{i}}\left(\bar{F}\left(V_{i} \mid Z_{i}\right)\right)^{1-\delta_{i}}=\prod_{i=1}^{n}\left(c_{i} f_{0}\left(V_{i}\right)\right)^{\delta_{i}}\left(\bar{F}_{0}\left(V_{i}\right)\right)^{c_{i}-\delta_{i}}
$$


Hence, the full likelihood function for $\left(\beta_{0}, F_{0}\right)$ in Cox model (1.1) with data (1.2) is given by

$$
L(\beta, F)=\prod_{i=1}^{n}\left(c_{i} d F\left(V_{i}\right)\right)^{\delta_{i}}\left(\bar{F}\left(V_{i}\right)\right)^{c_{i}-\delta_{i}}
$$

where $c_{i}=\exp \left(Z_{i} \beta\right)$. To profile out nuisance parameter $F_{0}$, we, without loss of generality, assume that there are no ties among $V_{i}$ 's with $V_{1}<\cdots<V_{n}$ (see Section 3 for treatment of ties), and we use the empirical likelihood parameterization (Owen, 1988; Shorack and Wellner, 1986). That is, we restrict all possible candidates for the MLE of $F_{0}$ to those d.f.'s that assign all their probability masses to points $V_{i}$ 's and interval $\left(V_{n}, \infty\right)$, which writes the full likelihood function (2.2) as follows:

$$
L(\beta, F)=\prod_{i=1}^{n}\left(c_{i} p_{i}\right)^{\delta_{i}}\left(\sum_{j=i+1}^{n+1} p_{j}\right)^{c_{i}-\delta_{i}}
$$

where $p_{i}=F\left(V_{i}\right)-F\left(V_{i}-\right)$ for $1 \leq i \leq n ; 0 \leq p_{n+1} \leq 1$ is the probability mass of d.f. $F$ on interval $\left(V_{n}, \infty\right)$; and we have $F(x)=\sum_{i=1}^{n} p_{i} I\left\{V_{i} \leq x\right\}$ satisfying $\sum_{i=1}^{n+1} p_{i}=1$.

Denoting $d_{i}=c_{i}+\cdots+c_{n}$, we show in the Appendix that for any fixed value $\beta$ satisfying $c_{n} \geq 1$, likelihood function $L(\beta, F)$ in $(2.3)$ is maximized by:

$$
1-\hat{F}_{n}(t)=\prod_{V_{i} \leq t} \frac{d_{i}-\delta_{i}}{d_{i}}
$$

In (2.3), we replace $F$ by $\hat{F}_{n}$, then from the proof of (2.4) given in the Appendix (see equation (A.1)), we obtain the following full-profile likelihood function for $\beta_{0}$ :

$$
\ell(\beta)=\prod_{i=1}^{n}\left(\frac{c_{i}}{d_{i}}\right)^{\delta_{i}}\left(\frac{d_{i}-\delta_{i}}{d_{i}}\right)^{d_{i}-\delta_{i}}=\ell_{c}(\beta) \prod_{i=1}^{n}\left(\frac{d_{i}-\delta_{i}}{d_{i}}\right)^{d_{i}-\delta_{i}}
$$

where $\ell_{c}(\beta)=\prod_{i=1}^{n}\left(c_{i} / d_{i}\right)^{\delta_{i}}$ is the Cox's partial likelihood function. Thus, the MLE for $\beta_{0}$ is given by the solution $\hat{\beta}_{n}$ which maximizes the value of $\ell(\beta)$, and consequently $\hat{F}_{n}$ in $(2.4)$ with $\beta$ replaced by $\hat{\beta}_{n}$ is the MLE for $F_{0}$.

Differentiating $\log \ell(\beta)$, algebra shows that $\hat{\beta}_{n}$ should be a solution of equation:

$$
\psi_{n}(\beta) \equiv n^{-1} \frac{d}{d \beta}(\log \ell(\beta))=n^{-1} \sum_{i=1}^{n} \delta_{i}\left(Z_{i}+e_{i} \log \frac{d_{i}-1}{d_{i}}\right)=0
$$

where $e_{i}=\sum_{j=i}^{n} Z_{j} c_{j}, \psi_{n}(\beta)$ is the full-profile likelihood estimating function, and from (2.5), $\log 0$ is set to 0 whenever it occurs. To compute $\hat{\beta}_{n}$, the Newton-Raphson method may be used with Cox's partial likelihood estimator $\hat{\beta}_{c}$ as the initial value. 
Remark 1. On Condition $c_{n} \geq 1$ : Throughout this section so far, all arguments require $c_{n} \geq 1$ for any fixed $\beta$, which ensures that for fixed $\beta$ : (a) Full likelihood function $L(\beta, F)$ in (2.3) has a finite maximum value; (b) $\hat{F}_{n}$ given by (2.4) maximizes $L(\beta, F)$ and is a proper d.f. with all terms on the right-hand side of equation between 0 and 1 , because $d_{i}>c_{n} \geq 1$ for all $1 \leq i<n$. These clearly are crucial reasons for having a well-defined full-profile likelihood function $\ell(\beta)$ as well as a well-defined MLE for $F_{0}$. Note that the requirement of $c_{n} \geq 1$ for any positive or negative $\beta$ is equivalent to requiring $Z_{n}=0$. Thus, in practice and for the rest of this paper, the natural way to handle this is to adjust $Z_{i}$ to $\tilde{Z}_{i}=Z_{i}-Z_{n}, 1 \leq i \leq n$, which rewrites model (1.1) as $\lambda\left(t ; Z_{i}\right)=\lambda_{\beta, n}(t) \exp \left(\tilde{Z}_{i} \beta\right)$ with $\lambda_{\beta, n}(t)=\lambda_{0}(t) \exp \left(Z_{n} \beta\right)$. In (2.3)-(2.6), we replace $Z_{i}$ and $c_{i}$ by $\tilde{Z}_{i}$ and $\tilde{c}_{i}=\exp \left(\tilde{Z}_{i} \beta\right)$, respectively, then we have $\tilde{c}_{n} \equiv 1$ for any $\beta$; the solution of (2.6) gives the MLE for $\beta_{0}$, still denoted as $\hat{\beta}_{n}$; and the resulting estimator in $(2.4)$, still denoted by $\overline{\hat{F}}_{n}$, is the MLE for $\left[\bar{F}_{0}(t)\right]^{z_{n} \beta}$, thus the MLE for $\bar{F}_{0}(t)$ is given by $\left[\overline{\hat{F}}_{n}(t)\right]^{e^{-Z_{n} \hat{\beta}_{n}}}$. Our extensive simulation studies show that such a treatment on condition $c_{n} \geq 1$ gives excellent performance on the resulting MLE $\hat{\beta}_{n}$ and the NewtonRaphson algorithm. In particular, $\tilde{c}_{n}=1$ for any positive or negative $\beta$ plays a crucial role for stable performance of the Newton-Raphson algorithm, because $\tilde{Z}_{i}$ 's in equation (2.6) ensure $\tilde{d}_{i}>1$ for all $1 \leq i<n$ and for any positive or negative $\beta$. Finally, we note that adjusting $Z_{i}$ to $\tilde{Z}_{i}=Z_{i}-Z_{n}, 1 \leq i \leq n$, does not change the Cox's partial likelihood function $\ell_{c}(\beta)$ in $(2.5)$, thus does not affect $\hat{\beta}_{c}$, because $\ell_{c}(\beta)=\prod_{i=1}^{n}\left(c_{i} / d_{i}\right)^{\delta_{i}}=\prod_{i=1}^{n}\left(\tilde{c}_{i} / \tilde{d}_{i}\right)^{\delta_{i}}$ and the partial likelihood estimating function in (1.3) satisfies

$$
\varphi_{n}(\beta)=n^{-1} \frac{d}{d \beta}\left(\log \ell_{c}(\beta)\right)=n^{-1} \sum_{i=1}^{n} \delta_{i}\left(Z_{i}-\frac{e_{i}}{d_{i}}\right)=n^{-1} \sum_{i=1}^{n} \delta_{i}\left(\tilde{Z}_{i}-\frac{\tilde{e}_{i}}{\tilde{d}_{i}}\right)
$$

where $\tilde{d}_{i}=\tilde{c}_{i}+\cdots+\tilde{c}_{n}$ and $\tilde{e}_{i}=\tilde{Z}_{i} \tilde{c}_{i}+\cdots+\tilde{Z}_{n} \tilde{c}_{n}$. The uniqueness of the MLE $\hat{\beta}_{n}$ and further rationale for using the given $\tilde{Z}_{i}$ is discussed in Remark 2.

Interestingly, by Taylor's expansion we show in the Appendix that the MLE $\hat{\beta}_{n}$ is linked with Cox's partial likelihood estimator $\hat{\beta}_{c}$ by the following:

$$
\psi_{n}(\beta)=\varphi_{n}(\beta)+O_{p}\left(\frac{\log n}{n}\right)
$$

where $\varphi_{n}(\beta)$ is the partial likelihood estimating function in (2.7). Further, Wilk's theorem on the log full-likelihood ratio is established below with proof given in the Appendix.

THEOREM 1. Assume (2.8) and assume the regularity conditions on Cox model (1.1) (Andersen and Gill, 1982). Then, $R_{0}=-2 \log \left[\ell\left(\beta_{0}\right) / \ell\left(\hat{\beta}_{n}\right)\right]$ converges in distribution to a chi-squared distribution with 1 degree of freedom as $n \rightarrow \infty$. 
Remark 2. Uniqueness of the $M L E \hat{\beta}_{n}$ : To further understand the rationale of using $\tilde{Z}_{i}=Z_{i}-Z_{n}, 1 \leq i \leq n$, as suggested in Remark 1 , we assume that the parameter space for $\beta$ satisfies $|\beta| \leq M_{1}<\infty$ for some constant $M_{1}$, and that the covariate variable $Z$ has a finite support. Then, there exists a constant $0<M<\infty$ such that we always have $\left|Z_{i} \beta\right| \leq M$ for $1 \leq i \leq n$ and any $|\beta| \leq M_{1}$, and we can rewrite Cox model (1.1) as $\lambda(t ; z)=\lambda_{M}(t) e^{z \beta+M}$ for $\lambda_{M}(t)=e^{-M} \lambda_{0}(t)$, which gives $C_{i}=e^{M} c_{i}=e^{Z_{i} \beta+M} \geq 1$ for all $1 \leq i \leq n$ and for any $|\beta| \leq M_{1}$. With these $C_{i}$ 's, the arguments in (2.3)-(2.5) show that equation (2.6) becomes $\psi_{M, n}(\beta) \equiv n^{-1} \sum_{i=1}^{n} \delta_{i}\left(Z_{i}+E_{i} \log \frac{D_{i}-1}{D_{i}}\right)=0$ with $D_{i}=\sum_{j=i}^{n} C_{j}$ and $E_{i}=\sum_{j=i}^{n} Z_{j} C_{j}$, the resulting estimator $\overline{\hat{F}}_{M, n}$ by equation (2.4) is the MLE for $\left[\bar{F}_{0}(t)\right]^{e^{-M}}$, and equation (2.8) holds for $\psi_{M, n}(\beta)$ for fixed $M$ as follows:

$$
\psi_{M, n}(\beta)=\varphi_{n}(\beta)+O_{p}\left(\frac{\log n}{n}\right), \quad \text { as } n \rightarrow \infty
$$

where $\varphi_{n}(\beta)$ is given by (2.7). The solution of equation $\psi_{M, n}(\beta)=0$ gives an MLE $\hat{\beta}_{M, n}$ for $\beta_{0}$. Thus, the MLE for $\beta_{0}$ depends on the choice of $M$; in turn, it is not unique due to the Cox model assumption (1.1) itself, which allows the use of an arbitrarily large enough constant $M$ in the way described above. On the other hand, by Taylor's expansion and a proof similar to that for (2.8), we can show that for any given survival sample (1.2),

$$
\psi_{M, n}(\beta)=\varphi_{n}(\beta)+o(1), \quad \text { as } M \rightarrow \infty
$$

where $o(1)$ is uniformly for $\beta$. Equations (2.9)-(2.10) together explain why the Cox's partial likelihood estimator $\hat{\beta}_{c}$ has the well-known good asymptotic properties, despite it is not the actual MLE. With (2.10) in mind, in practice we should choose $M$ considering the following two factors: (i) When $Z_{n} \beta \geq 0$, choose $M=0$, i.e., if $Z_{n} \beta \geq 0$, we have $c_{n} \geq 1$, thus there is no need to use $M$; (ii) When $Z_{n} \beta<0$, choose a small $M>0$ to have $C_{n} \geq 1$, because a large $M>0$ implies $\overline{\hat{F}}_{M, n}(t) \approx\left[\bar{F}_{0}(t)\right]^{e^{-M}} \approx 1$ and $\psi_{M, n}(\beta) \approx \varphi_{n}(\beta)$. Hence, in practice we use the smallest $M \geq 0$ under condition $C_{n}=e^{Z_{n} \beta+M} \geq 1$ for any $|\beta| \leq M_{1}$, and such an $M$ is given by $M_{n}=\min \left\{M \geq 0 \mid Z_{n} \beta+M \geq 0\right\}=-Z_{n} \beta I\left\{Z_{n} \beta<0\right\}$. Thus, for positive covariates $Z_{i}$ 's, we should use $M_{n}=0$ if $\beta_{0}>0 ; M_{n}=-Z_{n} \beta$ if $\beta_{0}<0$. Since in practice the true value of $\beta_{0}$ is unknown, we always use $M_{n}=-Z_{n} \beta$ (unless there is evidence for $Z_{n} \beta_{0}>0$ ), which gives $Z_{i} \beta+M_{n}=\tilde{Z}_{i} \beta$ as suggested in Remark 1. In conclusion, the MLE for $\beta_{0}$ in the Cox model (1.1) is generally not uniquely defined; but it is uniquely defined under assumptions: (a) we know nothing about the true value of $\beta_{0}$; (b) $c_{n}=e^{Z_{n} \beta} \geq 1$ for any positive or negative $\beta$ to ensure a well-defined full-profile likelihood function $\ell(\beta)$ in (2.5). And such unique MLE is given by the $\hat{\beta}_{n}$ in Remark 1 . Note that under Remark 1 , the MLE $\hat{\beta}_{n}$ is uniquely well defined as a solution of equation (2.6) with $\tilde{Z}_{i}$ 's in the places 
of $Z_{i}$ 's, and equation (2.8) and Theorem 1 hold with $\tilde{Z}_{i}$ 's in the places of $Z_{i}$ 's.

Remark 3. $p$-Dimensional $Z_{i}$ : If $Z_{i}$ and $\beta_{0}$ are $p$-dimensional vectors with $p>1$ in (1.1)-(1.2), with minor modifications in the derivations and proofs we have that (2.3)-(2.8) and Remarks 1-2 hold, where with $c_{i}=\exp \left(Z_{i}^{\top} \beta\right), e_{i}$ and $\tilde{Z}_{i}=Z_{i}-Z_{n}$ are $p$-dimensional vectors, and (2.6) has $p$ equations. Moreover, a minor modified proof of Theorem 1 shows that $R_{0}$ converges in distribution to a chi-squared distribution with $p$ degrees of freedom.

\section{Simulations and Treatment of Ties}

This section first presents some simulation results to compare the MLE $\hat{\beta}_{n}$ with Cox's partial likelihood estimator $\hat{\beta}_{c}$ for the case without ties among $V_{i}$ 's in (1.2). Then, we discuss how to handle ties among $V_{i}$ 's, and present some simulation results to compare $\hat{\beta}_{n}$ with Efron's estimator $\hat{\beta}_{E}$. In all our simulation studies, $\hat{\beta}_{n}$ is calculated using the NewtonRaphson method with $\hat{\beta}_{c}$ or $\hat{\beta}_{E}$ as the initial value. Routines in FORTRAN for computing $\hat{\beta}_{n}$ are available from the authors.

\section{Without Ties Among $V_{i}$ 's in (1.2):}

Let $\operatorname{Exp}(\mu)$ represent the exponential distribution with mean $\mu$, and $\mathrm{U}(0,1)$ the uniform distribution on interval $(0,1)$. In our simulation studies, we consider $F_{Y}=\operatorname{Exp}(2)$ as the d.f. of the right censoring variable $Y_{i}, F_{Z}=\mathrm{U}(0,1)$ as the d.f. of $Z$, and $F_{X \mid Z}=\operatorname{Exp}\left(e^{-Z \beta_{0}}\right)$ as the conditional d.f. of $X$ given $Z$; thus $(X, Z)$ satisfies the Cox model (1.1) with regression parameter $\beta_{0}$ and baseline d.f. $F_{0}=\operatorname{Exp}(1)$. For each case of $\beta_{0}=1,0,-1$, we generate 1000 samples with sample size $n=15,20,30,50$, respectively, and for each $n$ Table 1 includes the simulation average of $\hat{\beta}_{c}$ and $\hat{\beta}_{n}$ with the simulation standard deviation (s.d.) given in the parenthesis next to them, respectively. The censoring percentage in each case is also reported in Table 1.

Table 1. Comparison between $\hat{\boldsymbol{\beta}}_{c}$ and $\hat{\boldsymbol{\beta}}_{\boldsymbol{n}}$

\begin{tabular}{|c|c|c|c|c|c|c|}
\hline Parameter & \multicolumn{2}{|c|}{$\beta_{0}=1$} & \multicolumn{2}{c|}{$\beta_{0}=0$} & \multicolumn{2}{c|}{$\beta_{0}=-1$} \\
\hline Sample Size & Ave. $\hat{\beta}_{c}$ & Ave. $\hat{\beta}_{n}$ & Ave. $\hat{\beta}_{c}$ & Ave. $\hat{\beta}_{n}$ & Ave. $\hat{\beta}_{c}$ & Ave. $\hat{\beta}_{n}$ \\
\hline$n=15$ & $1.145(1.42)$ & $1.042(1.36)$ & $0.020(1.47)$ & $0.016(1.41)$ & $-1.120(1.72)$ & $-1.062(1.66)$ \\
$n=20$ & $1.116(1.13)$ & $1.035(1.09)$ & $0.052(1.23)$ & $0.047(1.16)$ & $-1.112(1.44)$ & $-1.060(1.39)$ \\
$n=30$ & $1.081(0.85)$ & $1.018(0.83)$ & $0.013(0.93)$ & $0.009(0.89)$ & $-1.066(1.04)$ & $-1.018(1.02)$ \\
$n=50$ & $1.036(0.64)$ & $0.988(0.63)$ & $0.003(0.68)$ & $-0.001(0.66)$ & $-1.020(0.74)$ & $-0.986(0.74)$ \\
\hline Censoring \% & \multicolumn{2}{|c|}{$23.7 \%$} & \multicolumn{3}{|c|}{$33.4 \%$} & \multicolumn{2}{c|}{$45.4 \%$} \\
\hline
\end{tabular}

Table 1 clearly shows that the MLE $\hat{\beta}_{n}$ performs better than Cox's partial likelihood estimator $\hat{\beta}_{c}$ for small or moderate sample sizes when $\beta_{0}$ is away from 0 . For instance, the loss in precision for $\beta_{0}= \pm 1$ with, say, $n=15$ is reflected by the simulation Mean Square Error (MSE). Simple calculation gives that when $\beta_{0}=1$, the simulation MSE is 
2.037 and 1.851 for $\hat{\beta}_{c}$ and $\hat{\beta}_{n}$, respectively, yielding $1.851 / 2.037=90.9 \%$ (such ratio is $92.2 \%$ for $n=20$ ), while when $\beta_{0}=-1$, the simulation MSE is 2.973 and 2.759 for $\hat{\beta}_{c}$ and $\hat{\beta}_{n}$, respectively, yielding $2.759 / 2.973=92.8 \%$ ( such ratio is $92.7 \%$ for $n=20$ ). The loss in precision for $\hat{\beta}_{c}$ shows even more obviously when we use $\beta_{0}= \pm 2, \pm 3, \cdots$ in simulation studies of Table 1 . To illustrate, we include results for $\beta_{0}=-2$ with sample size $n=15$ in Table 2 , where Relative Bias is $\left|\left(\hat{\beta}-\beta_{0}\right) / \beta_{0}\right|$, Relative MSE is $E\left[\left(\hat{\beta}-\beta_{0}\right) / \beta_{0}\right]^{2}$ and the censoring variable is still $\operatorname{Exp}(2)$. Note that in Table 2, the ratio of simulation relative MSE for $\hat{\beta}_{n}$ and $\hat{\beta}_{c}$ is $8.969 / 33.658=26.6 \%$. Also, note that with fixed censoring variable $\operatorname{Exp}(2)$, the censoring percentage increases as we choose a smaller and smaller $\beta_{0}$; thus our extensive simulation studies include cases with much higher censoring percentages than that shown in Table 2, and the results are similar to what's presented here.

Table 2. Comparison between $\hat{\boldsymbol{\beta}}_{c}$ and $\hat{\boldsymbol{\beta}}_{\boldsymbol{n}}$

\begin{tabular}{|c|c|c|c|}
\hline & \multicolumn{3}{|c|}{$\beta_{0}=-2, \quad n=15, \quad[$ Censoring Percentage $]=57.0 \%$} \\
\hline Estimator & Simulation Mean (s.d.) & Simulation Relative Bias & Simulation Relative MSE \\
\hline$\hat{\beta}_{c}$ & $-3.403(11.518)$ & 0.702 & 33.658 \\
$\hat{\beta}_{n}$ & $-2.828(5.932)$ & 0.414 & 8.969 \\
\hline
\end{tabular}

Finally, although not presented here, our simulation studies also show that the MLE $\hat{F}_{n}$ given in (2.4) provides a very good estimate for $1-\left[\bar{F}_{0}(t)\right]^{Z_{n \beta} \beta}$; see Remark 1.

\section{With Ties Among $V_{i}$ 's in (1.2):}

Let $W_{1}<\cdots<W_{m}$ be all the distinct observations of $V_{1} \leq \cdots \leq V_{n}$, where $m<n$, and for those tied $V_{j}$ 's, the uncensored $V_{j}$ 's are ranked ahead of the censored $V_{j}$ 's. If we have, say, $V_{1}=V_{2}=V_{3}=W_{1}$ with $\delta_{1}=\delta_{2}=1, \delta_{3}=0$, then by Efron's estimation (see pages 48-49; Therneau and Grambsch, 2000) $d_{i}$ 's in Cox's partial likelihood function $\ell_{c}(\beta)$ in $(2.5)$ are modified as $d_{1}=c_{1}+c_{2}+\cdots+c_{n}, d_{2}=\left(c_{1}+c_{2}\right) / 2+c_{3}+\cdots+c_{n}, d_{3}=c_{3}+\cdots+c_{n}$, etc., which give Efron's estimator $\hat{\beta}_{E}$. The idea of this Efron's estimation is that since the ties are caused by rounding errors in practice and we do not really know if it in fact is $V_{1}<V_{2}$ or $V_{2}<V_{1}$ (which affects the values of $d_{1}$ and $d_{2}$ ), we take 'average' to modify $d_{i}$ 's in $\ell_{c}(\beta)$; see Therneau and Grambsch (2000). In our full-profile likelihood function $\ell(\beta)$ given in (2.5), we face the same problem with $d_{i}$ 's when there are ties among $V_{i}$ 's; thus Efron's estimation for $d_{i}$ 's is applicable in $\ell(\beta)$ for the same reason as for $\ell_{c}(\beta)$. Hence, applying these Efron's modified $d_{i}$ 's in (2.5)-(2.6), the MLE for $\beta_{0}$ when there are ties among $V_{i}$ 's is given by the solution of (2.6), still denoted by $\hat{\beta}_{n}$.

Some simulation results are presented in Tables 3-4 to compare the MLE $\hat{\beta}_{n}$ with $\hat{\beta}_{E}$. In these simulation studies, we consider $n=15, F_{Y}=\operatorname{Exp}(2)$, and $F_{X \mid Z}=\operatorname{Exp}\left(e^{-Z \beta_{0}}\right)$, and we create ties among $V_{i}$ 's as follows: compute $t_{k}=V_{1}+\frac{k}{n}\left(V_{n}-V_{1}\right)$ for $0 \leq k \leq n+1$, 
and set $V_{i}=t_{k+1}$ if $V_{i} \in\left[t_{k}, t_{k+1}\right)$, which represents rounding errors in practice that cause tied values among $V_{i}$ 's. Table 3 includes the simulation results based on 1000 samples with $F_{Z}=\mathrm{U}(0,1)$ for $\beta_{0}=-2$ and $\beta_{0}=2$, respectively, and reports the average number $m$ of distinct $V_{i}$ 's. Table 4 includes results of the same simulation studies with $F_{Z}=\operatorname{Exp}(1)$ for $\beta_{0}=-0.75$ and $\beta_{0}=0.75$, respectively.

Table 3. Comparison between $\hat{\beta}_{E}$ and $\hat{\beta}_{n}$

\begin{tabular}{|c|c|c|c|c|c|}
\hline$F_{Z}=\mathrm{U}(0,1)$ & Estimator & Simul. Mean (s.d.) & Rel. Bias & Rel. MSE & Ave. $m$ \\
\hline$\beta_{0}=-2$ & $\hat{\beta}_{E}$ & $-3.025(9.044)$ & 0.513 & 20.711 & 8.3 \\
Censoring: $57.0 \%$ & $\hat{\beta}_{n}$ & $-2.662(5.541)$ & 0.331 & 7.785 & 8.3 \\
\hline$\beta_{0}=2$ & $\hat{\beta}_{E}$ & $2.167(1.349)$ & 0.084 & 0.462 & 7.8 \\
Censoring: $16.8 \%$ & $\hat{\beta}_{n}$ & $2.013(1.329)$ & 0.007 & 0.442 & 7.8 \\
\hline
\end{tabular}

Table 4. Comparison between $\hat{\boldsymbol{\beta}}_{E}$ and $\hat{\boldsymbol{\beta}}_{n}$

\begin{tabular}{|c|c|c|c|c|c|}
\hline$F_{Z}=\operatorname{Exp}(1)$ & Estimator & Simul. Mean (s.d.) & Rel. Bias & Rel. MSE & Ave. $m$ \\
\hline$\beta_{0}=-0.75$ & $\hat{\beta}_{E}$ & $-1.115(2.490)$ & 0.487 & 11.259 & 8.2 \\
Censoring: $50.4 \%$ & $\hat{\beta}_{n}$ & $-1.029(1.602)$ & 0.372 & 4.701 & 8.2 \\
\hline$\beta_{0}=0.75$ & $\hat{\beta}_{E}$ & $0.749(0.435)$ & 0.001 & 0.336 & 7.9 \\
Censoring: $20.9 \%$ & $\hat{\beta}_{n}$ & $0.713(0.428)$ & 0.049 & 0.328 & 7.9 \\
\hline
\end{tabular}

Overall, Tables 3-4 show that the MLE $\hat{\beta}_{n}$ performs favorably. In particular, note that in Table 3, the ratio of simulation relative MSE for $\hat{\beta}_{n}$ and $\hat{\beta}_{E}$ is $7.785 / 20.711=37.59 \%$ for $\beta_{0}=-2$, while in Table 4 , such ratio is $4.701 / 11.259=41.8 \%$ for $\beta_{0}=-0.75$.

\section{Data Example}

We consider the Stanford Heart Transplant data set (Escobar and Meeker, 1992; it is available in $\mathrm{R}$ library with file name 'stanford2'), where $Z$ is the age of a patient and $X$ is the survival time subject to right censoring. To see that different likelihood ratio tests can lead to different conclusions in practice for smaller data sets, we use observations number $76-100$ and observations number $50-100$, respectively, to test $\mathrm{H}_{0}: \beta_{0}=0$ vs. $\mathrm{H}_{1}: \beta_{0} \neq 0$ using Wald test, the partial likelihood ratio (PLR) test and our full likelihood ratio (FLR) test according to Theorem 1. The results are summarized in Table 5, which show that PLR test and FLR test have statistically different conclusions for sample size $n=25$ when $5 \%$ is used as the test significance level.

Of course, the point of this example is not to say that the FLR test is always more efficient than the PLR test; rather it demonstrates the fact that in practical situations FLR 
test and PLR test may sometimes lead to statistically different conclusions for small or moderate samples. This, along with the simulation results in Tables 1-4, indicates that the full-likelihood inferences for the Cox's model developed in this article are worth further examination and consideration.

Table 5. Stanford Heart Transplant Data

\begin{tabular}{|c|c|c|c|c|c|c|c|}
\hline & \multicolumn{2}{|c|}{ Data Information } & \multicolumn{2}{c|}{ Estimator } & \multicolumn{3}{c|}{$p$-value } \\
\hline Observations Used & $n$ & Censored Obs. & $\hat{\beta}_{c}$ & $\hat{\beta}_{n}$ & Wald Test & PLR Test & FLR Test \\
\hline No. $76-100$ & 25 & 8 & 0.367 & 0.397 & 0.063 & 0.056 & 0.038 \\
\hline No. $50-100$ & 51 & 23 & 0.153 & 0.149 & 0.050 & 0.045 & 0.049 \\
\hline
\end{tabular}

\section{Concluding Remarks}

We have derived the full likelihood function for $\left(\beta_{0}, F_{0}\right)$ in the Cox model (1.1) with right censored survival data (1.2), and have derived joint $\operatorname{MLE}\left(\hat{\beta}_{n}, \hat{F}_{n}\right)$ for $\left(\beta_{0}, F_{0}\right)$, where $\hat{F}_{n}$ is always a proper distribution function. While our simulation studies indicate that for small or moderate sample sizes the MLE $\hat{\beta}_{n}$ performs favorably over Cox's partial likelihood estimator $\hat{\beta}_{c}$, the theoretical estimation bias of our MLE $\hat{\beta}_{n}$ in comparison with that of the Cox's estimator $\hat{\beta}_{c}$ is to be investigated in a forthcoming paper.

A preliminary version of the $\mathrm{R}$-code that computes the multivariate $p$-dimensional MLE $\hat{\beta}_{n}$ and related quantities has been developed, and is now available at the following link: http://www.ms.uky.edu/ mai/Rcode/PHreg.fit.R.txt. Although the code needs to be further refined for publication quality, the computations we have conducted so far show that the algorithm is stable for the multivariate setting.

Acknowledgment. The authors thank the Associate Editor and two referees for their comments and suggestions on the earlier draft of this article.

\section{APPENDIX}

Proof of (2.4): Let $a_{i}=p_{i} / b_{i}$ and $b_{i}=\sum_{j=i}^{n+1} p_{j}$. Then, we have

$$
b_{1}=1, b_{n+1}=p_{n+1}, b_{i+1}=\left(b_{i}-p_{i}\right),\left(1-a_{i}\right)=b_{i+1} / b_{i} .
$$

From $\prod_{i=1}^{n}\left(1-a_{i}\right)=b_{n+1}$ and from

$$
\prod_{i=1}^{n}\left(a_{i}\right)^{c_{i}}\left(1-a_{i}\right)^{n-h_{i}}=\left(\prod_{i=1}^{n}\left(a_{i}\right)^{c_{i}}\right) \prod_{i=1}^{n}\left(\frac{b_{i+1}}{b_{i}}\right)^{n-h_{i}}=\left(\prod_{i=1}^{n}\left(a_{i}\right)^{c_{i}}\right)\left(b_{n+1}\right)^{n(1-\bar{c})} \prod_{i=1}^{n}\left(b_{i}\right)^{c_{i}},
$$


where $h_{i}=c_{1}+\cdots+c_{i}$ and $\bar{c}=n^{-1} \sum_{i=1}^{n} c_{i}$, we can rewrite $(2.3)$ as

$$
\begin{aligned}
L(\beta, F) & =\prod_{i=1}^{n}\left(c_{i} p_{i}\right)^{\delta_{i}}\left(b_{i}-p_{i}\right)^{c_{i}-\delta_{i}}=\left(\prod_{i=1}^{n}\left(c_{i}\right)^{\delta_{i}}\left(p_{i}\right)^{c_{i}}\right) \prod_{i=1}^{n}\left(\frac{1-a_{i}}{a_{i}}\right)^{c_{i}-\delta_{i}} \\
& =\left(\prod_{i=1}^{n}\left(c_{i}\right)^{\delta_{i}}\left(p_{i}\right)^{c_{i}}\right) \frac{\prod_{i=1}^{n}\left(a_{i}\right)^{\delta_{i}}\left(1-a_{i}\right)^{\left[n-\delta_{i}-\left(c_{1}+\cdots+c_{i-1}\right)\right]}}{\prod_{i=1}^{n}\left(a_{i}\right)^{c_{i}}\left(1-a_{i}\right)^{\left[n-\left(c_{1}+\cdots+c_{i}\right)\right]}} \\
& =\left(\prod_{i=1}^{n}\left(c_{i}\right)^{\delta_{i}}\left(p_{i}\right)^{c_{i}}\right) \frac{\prod_{i=1}^{n}\left(a_{i}\right)^{\delta_{i}}\left(1-a_{i}\right)^{\left[n-\delta_{i}-\left(c_{1}+\cdots+c_{i-1}\right)\right]}}{\left(b_{n+1}\right)^{n(1-\bar{c})} \prod_{i=1}^{n}\left(p_{i}\right)^{c_{i}}}=\prod_{i=1}^{n}\left(c_{i} a_{i}\right)^{\delta_{i}}\left(1-a_{i}\right)^{d_{i}-\delta_{i}} .
\end{aligned}
$$

From the 1 st and 2 nd partial derivatives of $\log L$ with respect to $a_{i}$ 's, we know that the solution of equations $\partial(\log L) / \partial a_{i}=0$ is given by $\hat{a}_{i}=\delta_{i} / d_{i}, 1 \leq i \leq n$, and it maximizes $L(\beta, F)$ under condition $c_{n} \geq 1$. Hence, (2.4) follows from noting that the $\hat{p}_{i}$ 's corresponding to $\hat{a}_{i}$ 's give $\overline{\hat{F}}_{n}(t)=\prod_{V_{i} \leq t}\left(1-\hat{a}_{i}\right)$ and that condition $c_{n} \geq 1$ implies all $0 \leq \hat{a}_{i} \leq 1$.

Proof of (2.8): From Remark 1, we give the proof for $\psi_{n}(\beta)$ in (2.6) with $\tilde{Z}_{i}=Z_{i}-Z_{n}, 1 \leq i \leq n$, assuming that $|\beta| \leq M_{\beta}<\infty$ and $Z$ has a finite support. From $\tilde{c}_{n}=1, \tilde{Z}_{n}=0$, equation (2.7) and Taylor's expansion, we have in (2.6),

$$
\psi_{n}(\beta)=n^{-1} \sum_{i=1}^{n} \delta_{i} \tilde{Z}_{i}-n^{-1} \sum_{i=1}^{n-1} \delta_{i} \tilde{e}_{i}\left(\frac{1}{\tilde{d}_{i}}+\frac{1}{2 \xi_{i}^{2}}\right)=\varphi_{n}(\beta)-\frac{1}{2} R_{n}
$$

where $R_{n}=n^{-1} \sum_{i=1}^{n-1}\left(\delta_{i} \tilde{e}_{i}\right) / \xi_{i}^{2}$ with $\xi_{i}$ between $\tilde{d}_{i}$ and $\left(\tilde{d}_{i}-1\right)$. From $\left(1-1 / \tilde{d}_{n-1}\right)^{-1}=$ $\left(1+1 / \tilde{c}_{n-1}\right)$ and $m_{i}(\beta)=\min \left\{\tilde{Z}_{j} \beta \mid i \leq j \leq n\right\}$, the proof follows from

$$
\begin{aligned}
\left|R_{n}\right| & \leq \frac{1}{n} \sum_{i=1}^{n-1} \frac{\left|\tilde{e}_{i}\right|}{\left(\tilde{d}_{i}-1\right)^{2}}=\frac{1}{n} \sum_{i=1}^{n-1} \frac{\left|\tilde{e}_{i}\right|}{\tilde{d}_{i}^{2}\left(1-1 / \tilde{d}_{i}\right)^{2}} \leq \frac{1}{n} \sum_{i=1}^{n-1} \frac{\left|\tilde{e}_{i}\right|}{\tilde{d}_{i}^{2}} \frac{1}{\left(1-1 / \tilde{d}_{n-1}\right)^{2}} \\
& \leq O_{p}\left(n^{-1}\right) \max _{1 \leq i \leq n}\left|\tilde{Z}_{i}\right| \sum_{i=1}^{n-1} \frac{\exp \left(-m_{i}(\beta)\right)}{\exp \left(\tilde{Z}_{i} \beta-m_{i}(\beta)\right)+\cdots+\exp \left(\tilde{Z}_{n} \beta-m_{i}(\beta)\right)} \\
& \leq O_{p}\left(n^{-1}\right) \sum_{i=1}^{n-1} \frac{1}{n-i+1}=O_{p}\left(\frac{\log n}{n}\right) .
\end{aligned}
$$

Proof of Theorem 1: From Remark 1, we give the proof for $\ell(\beta)$ in $(2.5)$ and $\psi_{n}(\beta)$ in (2.6) with $\tilde{Z}_{i}=Z_{i}-Z_{n}, 1 \leq i \leq n$, which implies $\tilde{c}_{n}=1$ and $\tilde{Z}_{n}=0$. Applying Taylor's expansion on $\log \ell\left(\beta_{0}\right)$ at point $\hat{\beta}_{n}$, we have that from $(2.6)$ and $\psi_{n}\left(\hat{\beta}_{n}\right)=0$,

$$
R_{0}=-n \psi_{n}^{\prime}(\xi)\left(\beta_{0}-\hat{\beta}_{n}\right)^{2}=-\psi_{n}^{\prime}(\xi)\left[\sqrt{n}\left(\hat{\beta}_{n}-\beta_{0}\right)\right]^{2}
$$

where $\xi$ is between $\hat{\beta}_{n}$ and $\beta_{0}$. From $(2.8)$, we know that $\sqrt{n}\left(\hat{\beta}_{n}-\beta_{0}\right)$ and $\sqrt{n}\left(\hat{\beta}_{c}-\beta_{0}\right)$ have 
the same limiting distribution $\mathrm{N}\left(0, \sigma^{2}\right)$ for some constant $0<\sigma^{2}<\infty$. It suffices to show that $-\psi_{n}^{\prime}(\xi)$ converges to $1 / \sigma^{2}$ in probability as $n \rightarrow \infty$.

From Taylor's expansion, $\tilde{c}_{n}=1, \tilde{Z}_{n}=0$ and (2.7), we have that in (2.6)

$$
\begin{aligned}
-\psi_{n}^{\prime}(\beta) & =-n^{-1} \sum_{i=1}^{n-1} \delta_{i}\left(\frac{d \tilde{e}_{i}}{d \beta} \log \frac{\tilde{d}_{i}-1}{\tilde{d}_{i}}+\frac{\tilde{e}_{i}^{2}}{\tilde{d}_{i}\left(\tilde{d}_{i}-1\right)}\right) \\
& =-n^{-1} \sum_{i=1}^{n-1} \delta_{i}\left\{\frac{d \tilde{e}_{i}}{d \beta}\left(-\frac{1}{\tilde{d}_{i}}-\frac{1}{2 \xi_{i}^{2}}\right)+\frac{\tilde{e}_{i}^{2}}{\tilde{d}_{i}}\left(\frac{1}{\tilde{d}_{i}}+\frac{1}{\eta_{i}^{2}}\right)\right\} \\
& =-\varphi_{n}^{\prime}(\beta)+\frac{1}{2} R_{1, n}-R_{2, n},
\end{aligned}
$$

where $R_{1, n}=n^{-1} \sum_{i=1}^{n-1} \delta_{i}\left(d \tilde{e}_{i} / d \beta\right) / \xi_{i}^{2}$ and $R_{2, n}=n^{-1} \sum_{i=1}^{n-1} \delta_{i} \tilde{e}_{i}^{2} /\left(\tilde{d}_{i} \eta_{i}^{2}\right)$ with $\xi_{i}$ and $\eta_{i}$ between $\tilde{d}_{i}$ and $\left(\tilde{d}_{i}-1\right)$. Applying the argument in (A.3) to $R_{1, n}$ and $R_{2, n}$, respectively, we obtain $-\psi_{n}^{\prime}(\beta)=-\varphi_{n}^{\prime}(\beta)+O_{p}((\log n) / n)$. The proof follows from the fact that $-\varphi_{n}^{\prime}(\beta)$ is the negative second derivative of the log of Cox's partial likelihood, and $-\varphi_{n}^{\prime}\left(\beta_{0}\right)$ converges to $1 / \sigma^{2}$ in probability as $n \rightarrow \infty$; see Andersen and Gill (1982).

\section{REFERENCES}

Andersen, P.K., Borgan, O., Gill, R.D., and Keiding, N.(1993). Statistical Models Based on Counting Processes. Springer-Verlag, New York.

Andersen, P.K. and Gill, R.D. (1982). Cox's regression model for counting processes: a large sample study. Ann. Statist. 10, 1100-1120.

Bailey, K.R. (1984). Asymptotic equivalence between the Cox estimator and the general ML estimators of regression and survival parameters in the Cox model. Ann. Statist. 12, 730-736.

Breslow, N.E. (1974). Covariance analysis of censored survival data. Biometrics 30, 89-99.

Chang, M.N. and Yang, G.L. (1987). Strong consistency of a nonparametric estimator of the survival function with doubly censored data. Ann. Statist. 15, 1536-1547.

Cox, D.R. (1972). Regression models and life-tables (with Discussion). J. R. Statist. Soc. B 34, 187-220.

Cox, D.R. and Oakes, D. (1984). Analysis of Survival Data. Chapman \& Hall, London.

Efron, B. (1977). The efficiency of Cox's likelihood function for censored data. J. Amer. Statist. Assoc. 72, 557-65.

Escobar, L.A. and Meeker, W.Q. (1992). Assessing influence in regression analysis with censored data. Biometrics 48, 507-528.

Fleming, T.R. and Harrington, D.P. (1991). Counting Processes and Survival Analysis. Wiley, New York.

Groeneboom, P. and Wellner, J.A. (1992). Information Bounds and Nonparametric Maximum likelihood Estimation. Birkhäuser Verlag. 
Gu, M.G. and Zhang, C.H. (1993). Asymptotic properties of self-consistent estimators based on doubly censored data. Ann. Statist. 21, 611-624.

Kalbfleisch, J.D. and Prentice, R.L. (1973). Marginal likelihoods based on Cox's regression and life model. Biometrika 60, 267-278.

Kalbfleisch, J.D. and Prentice, R.L. (2002). The Statistical Analysis of Failure Time Data. John Wiley \& Sons.

Kay, R. (1979). Some further asymptotic efficiency calculations for survival data regression models. Biometrika 66, 91-96.

Li, G., Li, R.Z. and Zhou, M. (2005). Empirical likelihood in survival analysis. Contemporary Multivariate Analysis and Design of Experiments, Edited by J. Fan and G. Li, 337-350. The World Scientific Publisher.

Murphy, S.A. and van der Vaart, A.W. (2000). On profile likelihood. J. Amer. Statist. Assoc. 95, 449-465.

Oakes, D. (1977). The asymptotic information in censored survival data. Biometrika 64, 441-448.

Owen, A.B. (1988). Empirical likelihood ratio confidence intervals for a single functional. Biometrika 75, 237-249.

Owen, A.B. (2001). Empirical Likelihood Chapman \& Hall, London.

Pan, X.R. (1997). Empirical Likelihood Ratio for Censored Data, Ph.D. Dissertation, Department of Statistics, University of Kentucky.

Shorack, G.R. and Wellner, J.A. (1986). Empirical Processes with Applications to Statistics. Wiley.

Therneau, T.M. and Grambsch, P.M. (2000). Modeling Survival Data, Extending the Cox Model. Springer.

Tsiatis, A.A. (1981). A large sample study of Cox's regression model. Ann. Statist. 9, 93-108. 